\title{
Generation and propagation of Alfvén waves in solar atmosphere
}

\author{
Yuri T. Tsap ${ }^{1,2}$, Alexander V. Stepanov ${ }^{2}$, and Yulia. G. Kopylova ${ }^{2}$ \\ ${ }^{1}$ Crimean Astrophysical Observatory, Nauchny, Crimea, Ukraine \\ email: yur@crao.crimea.ua \\ ${ }^{2}$ Central Astronomical Observatory at Pulkovo, Russia \\ email: stepanov@gao.spb.ru; yulia00@mail.ru
}

\begin{abstract}
The propagation of Alfvén waves from the photosphere into the corona with regard to the fine structure of the magnetic field is considered. The energy flux of Alfvén-type waves generated in the photosphere by convective motions does not depend on the ionization ratio. The reflection coefficient continuously decreases with a decrease of wave period. Influence of the external magnetic field on the Spruit cutoff frequency for transverse (kink) modes excited in the thin magnetic flux tubes is analyzed. Torsional modes can penetrate into the upper atmosphere most effectively since their amplitudes does not increase with height in the photosphere while kink ones can be transformed into shock waves in the lower chromosphere because of a significant increase of amplitudes. In spite of stratification the linearity of Alfvén-type modes in the chromosphere is conserved due to violation of the WKB approximation. The important role of the magnetic canopy is discussed. Alfvén waves generated by convective motions in the photosphere can contribute significantly to the heating of the coronal plasma in quite regions of the Sun.
\end{abstract}

Keywords. Sun: magnetohydrodynamics, waves, atmosphere, magnetic fields, corona

\section{Introduction}

It is currently believed that magnetohydrodynamic (MHD) waves (AC models), quasistationary electric currents or current sheets (DC models) can be responsible for the coronal heating (e.g., Narain \& Ulmshneider 1996). Since the role of nanoflares and microflares in the coronal heating is not clear the AC models proposed more than sixty years ago (e.g., Alfven 1947) remain relevant.

Alfvén waves, as distinguished from other MHD modes, do not compress plasma and they are considered as the main carrier of the energy of convective motions into the corona by many authors (e.g., Narain \& Ulmshneider 1996; Noble et al. 2003; Cranmer \& Ballegooijen 2005; Ofman 2005). However, the region of their generation is unknown; Alfvén waves can be excited in the convective zone, photosphere, chromosphere or corona.

Recently Vranjes et al. (2008) based on the MHD equations concluded that the generally accepted expression for estimates of energy fluxes of Alfvén waves $F=\rho \delta v_{0}^{2} / 2 v_{A}$, where $\delta v_{0}$ is the amplitude of the velocity perturbation, $\rho$ is the plasma density, $v_{A}=$ $B / \sqrt{4 \pi \rho}$ is the Alfvén speed, is unsuitable for the solar photosphere. They argued that due to the small ionization $\left(n_{i} / n_{a} \sim 10^{-4}\right)$ "the ion collisions do not feel the effects of the magnetic field". To our opinion, this inference is not valid since Vranjes et al. (2008) did not take into account electromagnetic forces in momentum equations (see next section).

Results, concerning the reflection of Alfvén waves, are very contradictory. For example, Thomas (1978) came to conclusion that these waves can not penetrate into the corona. Bel \& Leroy (1981) argued that energy flux, reaching the corona, is less than $10^{-5}$ of the input energy flux density for waves with periods $T_{p}=100-500 \mathrm{~s}$. On the other 
hand, according to Geronicolas (1977), reflection of Alfvén waves is negligible in the upper atmosphere of the Sun. Cranmer \& Ballegooijen (2005) concluded that waves are strongly reflected at the transition region and only about $5 \%$ of the wave energy can penetrate into the corona.

The urgency of these studies still increases in the light of results obtained by Noble et al. (2003). According to them, the propagation Alfvén-type waves (torsional and transverse) with $T_{p} \gtrsim 10 \mathrm{~s}$ in the isolated thin magnetic flux tubes is impossible in the solar chromosphere because of restrictions connected with the Spruit cutoff frequency. Though Musielak et al. (2007) have shown that Noble et al. (2003) made a mistake and the frequency cutoff for torsional modes can be neglected, nevertheless, as follows from some estimates (Noble et al. 2003), transverse modes are generated by convective motions more productively than torsional ones.

The coronal plasma density is tens and hundreds of millions of times lower than the photosphere one. As a result Alfvén modes should be transformed into the strongly dissipated shock waves (e.g., Hollweg 1982; Kudoh \& Shibata 1999) due to a sharp increase of amplitudes with height. Consequently, a question arises: can Alfvén waves penetrate from the photosphere into the corona of the Sun without significant energy losses?

\section{On the energy flux of Alfvén waves in the weakly ionized plasma}

Using the standard notation, the simplified momentum equations for electrons, ions, and neutrals can be written as

$$
\begin{gathered}
-e n_{e} \delta \mathbf{E}-\frac{e n_{e}}{c} \delta \mathbf{v}_{e} \times \mathbf{B}=0 \\
n_{i} M \frac{\partial \delta \mathbf{v}_{i}}{\partial t}=e n_{i} \delta \mathbf{E}+\frac{e n_{i}}{c} \delta \mathbf{v}_{i} \times \mathbf{B}+n_{i} M \nu_{i a}\left(\delta \mathbf{v}_{a}-\delta \mathbf{v}_{i}\right) \\
n_{a} M \frac{\partial \delta \mathbf{v}_{a}}{\partial t}=n_{a} M \nu_{a i}\left(\delta \mathbf{v}_{i}-\delta \mathbf{v}_{a}\right) .
\end{gathered}
$$

It should be emphasized that Vranjes et al. (2008) suggested that the role of Lorentz's force $f_{L}=\left|\delta \mathbf{v}_{i} \times \boldsymbol{\Omega}_{i}\right|$ is negligible on the right-hand side of equation (2) since $\Omega_{i} \ll \nu_{i a}$. However, the force caused by the ion drag $f_{i a}=\nu_{i a}\left|\delta \mathbf{v}_{i}-\delta \mathbf{v}_{a}\right| \rightarrow 0$ at $\delta \mathbf{v}_{a} \rightarrow \delta \mathbf{v}_{i}$ and the frequency $\nu_{i a}$ does not characterize the value of $f_{i a}$.

Taking into account that $n_{i}=n_{e}$ and current density $\mathbf{j}=e n_{i}\left(\delta \mathbf{v}_{i}-\delta \mathbf{v}_{e}\right)$, equations (1) and (2) give

$$
n_{i} M \frac{\partial \delta \mathbf{v}_{i}}{\partial t}=\frac{\delta \mathbf{j} \times \mathbf{B}}{c}+n_{i} M \nu_{i a}\left(\delta \mathbf{v}_{a}-\delta \mathbf{v}_{i}\right) .
$$

If the magnetic field $\mathbf{B} \| \mathbf{Z}$, the wave vector $\mathbf{k} \| \mathbf{B}$, and $\delta \mathbf{B}=\delta \mathbf{B}_{0} \exp (-i \omega t+i k z)$, then, adopting for the sake of simplicity $z=0$, equation (4), in view of Ampere's Law, $\delta \mathbf{j}=c / 4 \pi \nabla \times \delta \mathbf{B}$, takes the form

$$
\frac{\partial \delta v_{i}}{\partial t}=i b e^{-i \omega t}+\nu_{i a}\left(\delta v_{a}-\delta v_{i}\right), \quad b=\frac{k B \delta B_{0}}{4 \pi n_{i} M}
$$

where $\delta v_{i}$ and $\delta v_{a}$ are the transverse with respect to the magnetic field direction $\mathbf{B}$ components of the disturbed velocity.

Assuming $x=\delta v_{i}-\delta v_{a}$ and combing (3) and (5), we find

$$
\frac{\partial x}{\partial t}+\left(\nu_{i a}+\nu_{a i}\right) x=i b e^{-i \omega t} .
$$


Solution of equation (6) at $x(t=0)=x_{0}$ can be represented as

$$
x=\frac{i b e^{-i \omega t}}{\nu_{i a}+\nu_{a i}-i \omega}+\left(x_{0}-\frac{i b}{\nu_{i a}+\nu_{a i}-i \omega}\right) e^{-\left(\nu_{i a}+\nu_{a i}\right) t} .
$$

On the other hand, from equations (3) and (5), taking into account that

$$
n_{i} \nu_{i a}=n_{a} \nu_{a i}
$$

we have

$$
\frac{\partial \delta v_{i}}{\partial t}+\frac{\nu_{i a}}{\nu_{a i}} \frac{\partial \delta v_{a}}{\partial t}=i b e^{-i \omega t}
$$

Whence, assuming $y=\delta v_{i}+\nu_{i a} / \nu_{a i} \delta v_{a}$, we obtain

$$
\frac{\partial y}{\partial t}=i b e^{-i \omega t}
$$

Solution of last differential equation at $y(t=0)=y_{0}$ is reduced to the form

$$
y=y_{0}+\frac{b}{\omega}\left(1-e^{-i \omega t}\right)
$$

Thus, suggesting $\delta v_{0 a}=0, \delta v_{0 i}=-b / \omega, \omega \ll \nu_{a i}, t \gg 1 / \nu_{i a}$, and $n_{a} \gg n_{i}$, equations (7)-(9) give

$$
\delta v_{i} \approx \delta v_{a} \approx-\frac{n_{i}}{n_{a}} \frac{b}{\omega} e^{-i \omega t}
$$

At $\Omega_{i} \gg \omega$, taking $\delta \mathbf{v}=\delta \mathbf{v}_{i}$, from equations (2) and (10) we obtain the frozenin condition, i.e., $\delta \mathbf{E} \approx-\delta \mathbf{v} \times \mathbf{B} / c$. Since the averaging over a period the energy flux $\mathbf{F}=c / 8 \pi \delta \mathbf{E}^{*} \times \delta \mathbf{B}$, in view of (10) and the dispersion equation for Alfvén waves, $\omega=k v_{A}$, we get

$$
F_{z} \approx \frac{\delta B_{0}^{2}}{8 \pi} v_{A}
$$

It means, for instance, that in the solar photosphere, for which $\nu_{a i} \approx 10^{5} \mathrm{~s}^{-1}$ (Vranjes et al. 2008), the energy flux of waves $F_{z}$ with periods $T_{p} \gg 2 \pi / \nu_{a i} \approx 10^{-4}$ s does not depend on the degree of ionization. Equation (10) with the help of the energy conservation law, $\rho \delta v_{0}^{2} / 2 \approx \delta B_{0}^{2} / 8 \pi$, may be rewritten as $F_{z} \approx \rho \delta v_{0}^{2} / 2 v_{A}$, while as follows from Vranjes et al. (2008) the energy flux $F_{z} \approx \rho \delta v_{0 i}^{2} / 2\left(n_{i} / n_{a}\right) v_{A}$.

\section{On the reflection and propagation of Alfvén waves}

Model of the solar atmosphere. In terms of the structure of the magnetic field, we can divide the solar atmosphere into two parts: the region of the thin isolated magnetic flux tubes (photosphere-chromosphere) and the region of the quasi-homogeneous magnetic field (chromosphere-corona). The boundary between these regions is called the magnetic canopy. Based on this simple model we shall consider the propagation of Alfvén waves from the photosphere into the corona.

Reflection of Alfvén waves. The wave equation for Alfvén waves in the stratified atmosphere at $B=$ const can be represented as (e.g., Tsap 2006)

$$
\frac{\partial^{2} \delta v_{\perp}}{\partial t^{2}}=v_{A}^{2}(z) \frac{\partial^{2} \delta v_{\perp}}{\partial z^{2}} .
$$

At $v_{A} \propto \exp (z / 2 H)$ and $\delta v, \delta B \propto \exp (-i \omega t)$ the solution of equation (11) is

$$
\delta v_{\perp}=\left[C_{1} H_{0}^{(1)}(\eta)+C_{2} H_{0}^{(2)}(\eta)\right] e^{i \omega t},
$$


where $C_{1}$ and $C_{2}$ are arbitrary constants, $\eta=2 H \omega / v_{A}, H_{0}^{(1)}$ and $H_{0}^{(2)}$ are the Hankel functions, which describe waves propagating in different directions.

Ferraro \& Plumpton (1958), taking into account that Macdonald function $N_{0}(\eta) \rightarrow$ $-\infty$ at $\eta \rightarrow 0$, wrote the solution of equation (11) in the upper solar atmosphere as

$$
\delta v_{\perp}=C_{3} J_{0}(\eta) .
$$

Last equation describes oscillations, which cannot transfer the wave energy since the energy flux is equal to zero in this case. As a result, for example, Thomas (1978) came to conclusion about the total reflection of Alfvén waves in the upper atmosphere of the Sun. An et al. (1989) for justification of this approach used a "paradox" connected with the value of Alfvén velocity $\left(v_{A} \rightarrow \infty\right.$ at $\left.\rho \rightarrow 0\right)$. However, if we take into account the displacement current, the dispersion relation of Alfvén waves at $\omega \ll \Omega_{i}$ takes a form: $\omega / k=c / \sqrt{1+4 \pi \rho c^{2} / B^{2}}$. Therefore $\omega / k \rightarrow c$ at $\rho \rightarrow 0$, i.e., Alfvén waves are transformed into electromagnetic ones. Moreover, this suggests that the idea of the continues reflection of Alfvén waves in the stratified atmosphere (Bel \& Leroy 1981; An et al. 1990; Musielak \& Moore 1995) is not sufficiently correct.

There are many indications that the transmission of Alfvfen waves into the corona is determined by the reflection in the transition region (see, e.g., Schwartz et al. 1984; Cranmer \& Ballegooijen 2005). According to estimates, obtained by Tsap (2006), under conditions of the solar atmosphere the transmission coefficient for waves with periods less than few tens of seconds is about 0.3. It means that Alfvén modes with $T_{p}=10-40 \mathrm{~s}$ (waves with $T_{p}<10 \mathrm{~s}$ are strongly damped in the solar chromosphere due to ion-neutral collisions) can effectively penetrate into the corona.

Equilibrium condition for a thin magnetic flux tube. If a vertical magnetic flux tube is thin, the condition of equilibrium along the vertical axis $Z$ at the temperature $T(z)=$ const is

$$
p(z)=p(0) e^{-z / H} .
$$

The balance of pressures at the tube boundary reduces to equality

$$
p_{i}(z)+\frac{B_{i}^{2}(z)}{8 \pi}=p_{e}(z)+\frac{B_{e}^{2}(z)}{8 \pi} .
$$

In particular, for $T_{i}(z)=T_{e}(z)=$ const and $B_{i}(z) \propto B_{e}(z)$ or $B_{e}(z)=0$, we have from (13) and (14)

$$
e^{-z / H}=\frac{B_{i}^{2}(z)}{B_{i}^{2}(0)}=\frac{B_{e}^{2}(z)}{B_{e}^{2}(0)},
$$

i.e., $v_{A}^{2}(z)=$ const. Consequently, Alfvén velocity both inside and outside of a magnetic flux tube must be constant.

Transverse waves and the generalized Spruit cutoff frequency. As distinguished from Spruit (1981) we consider transverse oscillations of the thin magnetic flux tubes surrounded by the magnetic field $B_{e}$. The force related to the response of the external medium we write as follows

$$
\mathbf{f}_{\perp}=-\rho_{e} \frac{\partial \delta \mathbf{v}_{\perp}}{\partial t}+\frac{B_{e}^{2}}{4 \pi} \mathbf{c},
$$

where $\mathbf{c}=\partial \mathbf{e}_{l} / \partial l$ is the vector of curvature of perturbed magnetic field lines, $\mathbf{e}_{l}$ is the unit vector directed along the magnetic field. If $\delta \mathbf{v}_{\perp}=\delta v_{x}, \mathbf{g}=-g \mathbf{e}_{z}$, the equation, describing transverse Alfvén modes of a thin magnetic flux tube in the stratified atmosphere, can 
be represented as follows

$$
\left(\rho_{i}+\rho_{e}\right) \frac{\partial^{2} \delta v_{x}}{\partial t^{2}}-\frac{B_{i}^{2}+B_{e}^{2}}{4 \pi} \frac{\partial^{2} \delta v_{x}}{\partial z^{2}}+\left(\rho_{i}-\rho_{e}\right) g \frac{\partial \delta v_{x}}{\partial z}=0 .
$$

Note that equation (16) takes the form of the wave equation of torsion waves at $\delta v_{x}=\delta v_{\varphi}$, $B_{e}=0$, and $\rho_{e}=\rho_{i}$ (see, e.g., Noble et al. 2003)

$$
\frac{\partial^{2} \delta v_{\varphi}}{\partial t^{2}}=v_{A i}^{2} \frac{\partial^{2} \delta v_{\varphi}}{\partial z^{2}}
$$

Using (13)-(15), for transverse waves, instead of (16), we have

$$
v_{k}^{2} \frac{\partial^{2} \delta v_{x}}{\partial z^{2}}-\frac{\Delta_{B}}{2 H} \frac{\partial \delta v_{x}}{\partial z}+\omega^{2} \delta v_{x}=0
$$

where

$$
v_{k}^{2}=\frac{B_{i}^{2}+B_{e}^{2}}{4 \pi\left(\rho_{i}+\rho_{e}\right)}=\text { const, } \Delta_{B}=\frac{B_{i}^{2}-B_{e}^{2}}{4 \pi\left(\rho_{i}+\rho_{e}\right)}=\text { const. }
$$

Solution of equation (18) is

$$
\delta v_{x}=e^{\Delta_{B} z /\left(4 v_{k}^{2} H\right)}\left(C_{1} e^{i \kappa z}+C_{2} e^{-i \kappa z}\right), \quad \kappa=\frac{1}{2} \sqrt{\left(\frac{2 \omega}{v_{k}}\right)^{2}-\left(\frac{\Delta_{B}}{2 v_{k}^{2} H}\right)^{2}},
$$

i.e, the generalized Spruit cutoff frequency is equal to $\Omega_{A}=\Delta_{B} /\left(4 v_{k} H\right)$. When the plasma parameter $\beta<1$, in view of (14), we can accept $B_{i} \approx B_{e}$, therefore we obtain the amplitude $\left|\delta v_{x}(z)\right|=$ const and frequency $\Omega_{A} \approx 0$ from (19). Meanwhile, if a magnetic tube is isolated $\left(B_{e}=0\right)$, then, in contrast to the previous case, the wave amplitude $\delta v_{x}$ must increase significantly with height. It means that the external magnetic field not only suppresses the growth of amplitudes of transverse waves in the isothermal atmosphere but also considerably reduces the Spruit cutoff frequency $\Omega_{A}$.

It is interesting to note that for $\mathbf{B}=$ const, $\Delta_{B}=0$, and $\rho_{i}=\rho_{e}$ equation (16) is transformed to the expression, which coincides with the wave equation (11), describing Alfvén waves in the stratified atmosphere with the quasi-homogeneous magnetic field.

Amplitudes of Alfvén-type modes in the stratified atmosphere. Let us consider the variation of amplitudes of transverse and torsional modes above and below the magnetic canopy, suggesting that the wave generation takes place in the photosphere of the Sun.

As follows from equation (19), the expression connected amplitudes of transverse waves of the isolated magnetic flux tubes $\left|\delta v_{p}\right|$ and $\left|\delta v_{h}\right|$ at the photospheric level $z=0$ and some given height $z$, respectively, takes the form

$$
\frac{|\delta v(z)|}{|\delta v(0)|}=\left(\frac{n(0)}{n(z)}\right)^{1 / 4}
$$

If we use for the solar atmosphere the VAL $\mathrm{C}$ model and assume number densities $n(0) \approx$ $10^{17} \mathrm{~cm}^{-3}$ and $n(z)=10^{14} \mathrm{~cm}^{-3}(\approx 700 \mathrm{~km})$, from $(20)$ we obtain $|\delta v(z)| /|\delta v(0)| \approx 6$. Thus, nonlinear effects can play an important role even in the lower chromosphere for transverse waves. In contrast, according to (17), amplitudes of torsional waves do not depend on the height $z$ since the internal Alfvén velocity $v_{A i}=$ const.

Let us now estimate the growth of amplitudes in the region above the magnetic canopy, where magnetic field is quasi-homogeneous and the waves are described by equation (11). Adopting $v_{A}=v_{A 0} \exp \left[\left(z-z_{0}\right) / 2 H\right]$, where $z_{0}$ is the initial height, using (12), we derive

$$
\frac{|\delta v(z)|}{v_{A}(z)}=\frac{\left|\delta v\left(z_{0}\right)\right|}{v_{A}\left(z_{0}\right)} \sqrt{\frac{J_{0}^{2}(\eta)+N_{0}^{2}(\eta)}{J_{0}^{2}\left(\eta_{0}\right)+N_{0}^{2}\left(\eta_{0}\right)}} e^{-\left(z-z_{0}\right) / 2 H} .
$$


Numerical analysis of equation (21) shows that the relative amplitudes $|\delta v(z)| / v_{A}(z) \mid$ decrease with height $z$. In turn, since the disturbed magnetic field $\delta B \propto \partial \delta v / \partial z$, it is easy to make sure that the relative amplitudes $|\delta B(z)| / B\left(z_{0}\right)$ decrease with height as well.

\section{Conclusions}

In this work we have obtained the following results:

(a) The energy flux of Alfvén waves with periods $T_{p} \gg 10^{-4} \mathrm{~s}$ does not depend on the degree of ionization in the solar photosphere.

(b) The energetic losses of linear waves with periods less than several tens of seconds, propagating from the solar photosphere to the corona, are about $70 \%$ due to reflection.

(c) The external magnetic field of the thin magnetic flux tubes slows down with height the growth of amplitudes of transverse waves as well as decreases the Spruit frequency.

(d) The capability of transverse waves to heat coronal plasma depends on the height of the formation of the magnetic canopy.

(e) Torsional waves transfer the energy of convective plasma motions on the Sun and stars from the photosphere to the corona more efficiently than transverse ones since their amplitudes in the thin magnetic flux tubes are not changed.

\section{Acknowledgements}

We would like to thank the anonymous referee for helpful comments and suggestions. This work was partially supported by the Russian Foundation for Basic Research (projects No 06-02-16859-a, 09-02-00624-a), the Program of Presidium of Russian Academy of Sciences "Solar Activity and Physical Processes in the Sun-Earth System", the Program of the Department of Physical Sciences of RAS "Study of Solar-Wind Disturbance Sources" and the Program for Support of Leading Scientific Schools (NSh-6110.2008.2).

\section{References}

Alfven, H. 1947, MNRAS, 107, 211.

An, C.-H., Musielak, Z. E., Moore, R. L., \& Suess, S. T. 1989, ApJ, 345, 597.

An, C.-H., Suess, S. T., Moore, R. L., Musielak, Z. E. 1990, ApJ, 350, 309.

Bel, N. \& Leroy, B. 1981, Astron. Astrophys., 104, 203.

Cranmer, S. R. \& van Ballegooijen, A. A. 2005, ApJS, 156, 265.

Ferraro, V. C. A. \& Plumpton, C. 1958, ApJ, 127, 459.

Geronicolas, E. A. 1977, ApJ, 211, 966.

Hollweg, J. V. 1982, ApJ, 254, 806.

Kudoh, T. \& Shibata, K. 1999, ApJ, 514, 493.

Musielak, Z. E. \& Moore, R. L. 1995, ApJ, 452, 434.

Musielak, Z. E., Routh, S., \& Hammer R. 2007, ApJ, 659, 650.

Narain, U. \& Ulmshneider, P. 1996, Space Sci. Revs, 75, 453.

Noble, M. W., Musielak, Z. E., \& Ulmschneider P. U. 2003, Astron. Astrophys., 409, 1085.

Ofman, L. 2005, Space Sci. Revs, 120, 67.

Schwartz, S. J., Cally, P. S., \& Bel, N. 1984, Solar Phys., 92, 81.

Spruit, H. C. 1981, Astron. Astrophys., 98, 155.

Thomas, J. H. 1978, ApJ, 225, 275.

Tsap, Y. T. 2006, in Proccedings IAU Symposium No. 233, p.253.

Vranjes, J., Poedts, S., Pandey, B. P., \& de Pontieu, B. 2008, Astron. Astrophys., 478, 553. 


\section{Discussion}

GABRIEL: Your estimate of $10 \%$ wave reflection should still allow sufficient Alfven wave energy flux to penetrate and heat the corona, since it is estimated that there is large wave energy flux in the chromosphere and below (for example, see DePontieu et al., Science, 2007).

TSAP: You are right. However, according to our calculations the period of Alfven waves must be less than $40 \mathrm{~s}$. This follows from the expression for the reflection coefficient, which is different from the formula obtained by Hollweg (1984). 